\title{
Application of Fiber Bragg Gratings to Wearable Garments
}

\author{
João Paulo Carmo, Member, IEEE, Alexandre Manuel Ferreira da Silva, Student Member, IEEE, \\ Rui Pedro Rocha, and José Higino Correia, Member, IEEE
}

\begin{abstract}
This paper presents a photonic system based on Fiber Bragg Gratings (FBGs) for application to wearable garments. The objective is spanning the FBGs over the whole area of the garments for acting as sensing elements. The FBGs are embedded on a polychloroethanediyl (polyvinyl chloride, commonly abbreviated as PVC) carrier in order to increase their sensitivity to strains and for improving the simultaneous acquisition of respiratory and cardiac frequencies with only one FBG sensor. The global structure comprising FBGs and carrier allow high strain cycles and at the same time present linear behavior with the temperature, $17 \mathrm{pm} \cdot{ }^{\circ} \mathrm{C}^{-1}$. The measurements show a stable structure for temperatures up to $100^{\circ} \mathrm{C}$. This brings excellent perspectives for measuring the temperature with high accuracy and range. A set of tests were done by subjecting the FBG/carrier structure (with FBG stretched and no curves) to strains up to $1.2 \mathrm{~mm}$, and it was also observed a linear behaviour: e.g., displacements of $0.8 \mathrm{pm} \cdot \mu \varepsilon^{-1}$. Behind its sensing enhancement operation, the carrier makes easy to mount the sensing structures.
\end{abstract}

Index Terms-Fiber Bragg grating (FBG), noninvasive monitoring, optical fiber sensors, wearable devices.

\section{INTRODUCTION}

I $\mathrm{T}$ IS NOT anymore strange the link between textiles and electronics, where the combination of electronics and textiles into a single structure in order to obtain electronic textiles or e-textiles [1]. In this context, this paper presents a photonic system based on Fiber Bragg Grating (FBG) for integration in garments used on environments infested with high-intensity radiation or subjected to high-magnetic fields. Solutions supported in photonic systems are good ways due to its robustness and resilience when subjected to the former conditions [2]. Optical fibers are good ways for achieving such measurement goal [3]. The first set of tries to integrate optical fibers on textiles was done in the Georgia Tech's wearable motherboard (GTWM, also known as smart shirt), where the objective of the fibers was in the bullet wounds detection for soldiers acting on war scenarios [4]. The previous works integrating FBGs into textiles pushed this concept further,

Manuscript received June 28th, 2011; revised May 18, 2011; accepted June 28, 2011. Date of publication July 05, 2011; date of current version December 01, 2011. This work was supported by the Foundation for Science and Technology FCT/MIT-PT/EDAM-SI/0025/2008 project. The associate editor coordinating the review of this paper and approving it for publication was Prof. Ignacio Matias.

The authors are with the Department of Industrial Electronics, University of Minho, Campus Azurém, 4800-058 Guimarães, Portugal (e-mail: jcarmo@dei.uminho.pt; id1894@alunos.uminho.pt; rrocha@dei.uminho.pt; higino.correia@dei.uminho.pt).

Color versions of one or more of the figures in this paper are available online at http://ieeexplore.ieee.org.

Digital Object Identifier 10.1109/JSEN.2011.2161281 by presenting continuous monitoring systems for healthcare monitoring applications. The work developed by Jonckheere et al. [5], [6] is one of the first known approaches integrating FBGs in textiles and enables the measurement of respiratory movements under magnetic resonance imaging environments. A detailed comparison between three methods (all integrating fibers into textiles, e.g., macrobending sensors, FBG sensors, and time reflectometry sensors) for measuring the elongation of the abdominal circumference during breathing movements is analyzed by Grillet et al. [7]. The photonic system presented in this paper presents a complete monitoring system based on a single FBG concept for sensing both the respiratory and the cardiac frequency, as well as the environment temperature.

\section{FBG-BASED PHOtoniC System}

\section{A. FBG Sensors}

FBG sensors can find wide applications on telecommunication and sensing systems. In fact, the FBGs are suitable sensing elements for doing physical measurements where a kind of displacement is available. Examples of such applications are found in the literature include the measurements of strain [8], tilt rotation by an angle [9], temperature [10] humidity [11], and magnetic fields [12]. FBGs are periodic changes in the refraction index of the fiber core made by adequately exposing the fiber to intense ultraviolet (UV) light. The resonance behavior depends on grating pitch and on their axial variation because the resonance behavior strictly follows external actions in the exact proportion as the silica matrix surrounding the gratings. The ultimate effect of the resonances is the reflection by successive and coherent scatterings from the index modulation of a narrowband of the incident optical field injected into the fiber. In a FBG, the strongest interaction or mode-coupling responsible for the reflected light occurs at a well-known wavelength, $\lambda_{B}[\mathrm{~nm}]$, also known as Bragg wavelength. The Bragg's spectral component depends directly on the grating period of the FBG, $\Lambda$ [nm], on the modal index, $n_{\text {eff }}$, also known as effective refractive index of the FBG, and is given by [13]

$$
\lambda_{B}=2 n_{\mathrm{eff}} \Lambda \text {. }
$$

The shift in the wavelength, $\Delta \lambda_{B}[\mathrm{~nm}]$, with respect to the cross-sensitivity with the temperature and the axial strain change, $\Delta T[\mathrm{~K}]$ and $\Delta \varepsilon$, respectively, is given by [13], [14]

$$
\frac{\Delta \lambda_{B}}{\lambda_{B}}=\left(1-\rho_{\varepsilon}\right) \Delta \varepsilon+(\alpha+\xi) \Delta T
$$

where $\rho_{\varepsilon}$ is the photo-elastic coefficient of the fiber, $\alpha$ is the thermal expansion coefficient of the fiber material, and $\xi$ is the thermo-optic coefficient of the fiber material. For a $1550 \mathrm{~nm}$ 


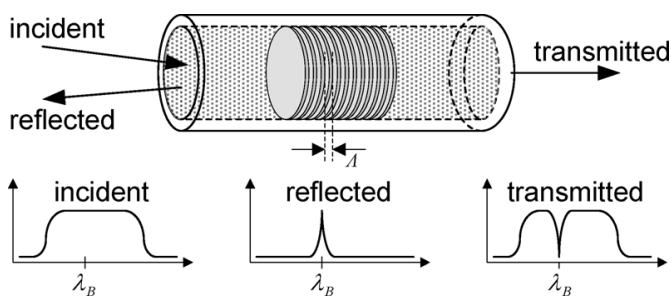

Fig. 1. Illustration of working principle of FBGs.

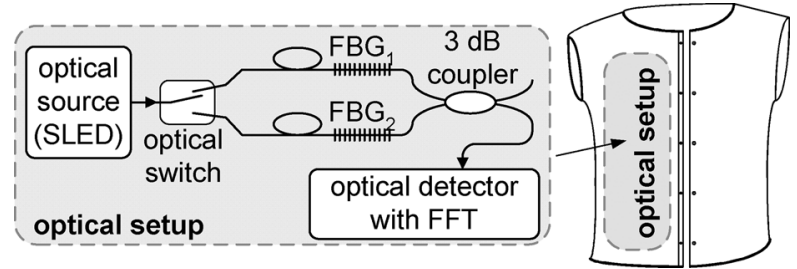

Fig. 2. The block diagram of the complete monitoring system comprising two channels tuned to a single- $\lambda$ by way of a single FBG.

FBG inscribed into a silica fiber, the wavelength sensitivities with respect to changes in the temperature and in the axial strain are of $\approx 13 \mathrm{pm} \cdot{ }^{\circ} \mathrm{C}^{-1}$ and $\approx 1 \mathrm{pm} \cdot \mu \varepsilon^{-1}$, respectively [13]. It must be noted that for only-strain measuring, the temperatureoffset compensation is mandatory to do.

Fig. 1 illustrates the FBG structure which is written on an optical fiber. This figure also illustrates that the injection of a broadband- $\lambda$ pulse on the FBG results in a reflected beam located around the Bragg wavelength, $\lambda_{B}$ [15]. It is possible to determine the exact strain by measuring the reflected spectra and/or the shifting produced in the Bragg wavelength [14]. Since the transmitted beam is the complementary of the reflected one, it is also possible to measure the exact strain [16]. The next section will be discussed the reasons for selecting this interrogation method.

\section{B. FBG Interrogation System}

In the system presented in this paper, a single FBG was used for simultaneously sensing the respiratory and cardiac frequencies, as well as the environment temperature. As illustrated in the Fig. 2, both $\mathrm{FBG}_{1}$ and $\mathrm{FBG}_{2}$ are tuned for the same wavelength, $\lambda[\mathrm{nm}]$, and demultiplexed with the help of a MEMS optical switch.

The proposed system is composed by three main subsystems: optical source (e.g., SLED), all-optical interrogation system (e.g., $2 \times 2$ fiber coupler, optical switch and the optical fibers), and the optical detector (with spectral analysis feature).

The optical source is a DL-CS5107A series SLED (Superluminiscent LED) operating in a true inherent superluminescent mode in order to generate a broadband light-beam and is fabricated by DenseLight Semiconductors Company [17]. This light source can generate a broadband light beam (in $[1530,1570] \mathrm{nm}$ range) with a maximal power of $8 \mathrm{~mW}$.

The used FBGs were produced by the FiberSensing Company [18]. The selected length for the gratings was $8 \mathrm{~mm}$ and the resonance wavelength was tuned for $1541 \mathrm{~nm}$, which corresponds to a refraction index modulation period of the core in the half-micrometer range $(\approx 0.52 \mu \mathrm{m})$ based on the effective refractive index of 1.47 .
TABLE I

THE Used COMPONENTS IN THE PHOTONIC SysteM

\begin{tabular}{|c|c|c|c|}
\hline Component & Weight & Size dimensions & $\begin{array}{c}\text { Power } \\
\text { consumption }\end{array}$ \\
\hline $\begin{array}{c}\text { Optical source } \\
\text { (SLED) }\end{array}$ & & $40 \times 30 \times 9 \mathrm{~mm}^{3}$ & $875 \mathrm{~mW}$ \\
\hline $2 \times 2$ coupler & & $66 \mathrm{~mm} \times \phi 3.8 \mathrm{~mm}$ & \\
\hline $1 \times 2$ optical switch & $94 \mathrm{~g}$ & $40 \times 50 \times 15 \mathrm{~mm}^{3}$ & \\
\hline FBGs+connectors & & & $150 \mathrm{~mW}$ \\
\hline Optical detector & $150 \mathrm{~g}$ & $70 \times 46 \times 18 \mathrm{~mm}^{3}$ & \\
\hline
\end{tabular}

The optical switch model EK703-FC from Thorlabs GmbH [19] was selected for demultiplexing two optical fiber Bragg gratings. This component is a MEMS $1 \times 2$ fiber optical switch for use in the $[1520,1610] \mathrm{nm}$ wavelength range, which contains an electronic system that controls a MEMS-actuated mirror for selecting the FBG to measure. This optical switch presents other characteristics that make it suitable for the proposed measuring application, e.g., low insertion loss (at $1550 \mathrm{~nm}$ ) of $0.7 \mathrm{~dB}$, high crosstalk rejection of $50 \mathrm{~dB}$, and capability to handle high optical powers (up to $300 \mathrm{~mW}$ ). Also, it is not necessary to use an optical isolator after the optical source due to the high return loss of $65 \mathrm{~dB}$. The optical switch contains a digital input for selecting the output channel. Since the voltage of this signal can change between 0 and $5 \mathrm{~V}$, the switch can be controlled by a microcontroller.

The 10202A-50 single-mode $2 \times 2$ fiber-optic coupler with a split ratio of 50/50 [\%] from Thorlabs GmbH [19] was selected in order to combine the readings from the two optical fibers with FBGs. The most used method to get the readings from FBGs is by reflection and not by transmission. However, the reflection method implies the use of more complex solutions. The first one requires the use of an optical circulator for separating the incident and the reflected beams from the respective ports and a bidirectional switch. The second most complex solution do not require the use of an optical circulator but instead needs three fiber-optic couplers to provide the readings to the optical detector (one for each fiber between the FBG and the respective output port of the switch, and a third to combine the two reverse outputs of the previous two couplers). As illustrated in Fig. 2, it is possible to combine the two optical fibers with FBGs by using only one single optical fiber coupler and thus, achieving a cheaper and simpler solution.

Finally, the optical detector is an I-MON 80D interrogation monitor from Ibsen Photonics [20] which is especially suitable for real-time spectrum monitoring of signals acquired from $\mathrm{FBG}$ sensors. The operating temperature range of $0{ }^{\circ} \mathrm{C}-70{ }^{\circ} \mathrm{C}$, the $70 \times 46 \times 18 \mathrm{~mm}^{3}$ dimensions, and the low weight of $150 \mathrm{~g}$ makes this optical detector appropriated for integration in wearables uses in extreme harsh environments.

Table I shows the weight, size and power consumptions of the individual components. The shaded cells refer to blocks with negligible weight when compared with most bulk blocks (e.g., the optical switch and detector). The measurements were done using the SLED as optical source, which requires a source with a regulated voltage of $2.5 \mathrm{~V}$ and a power of $875 \mathrm{~mW}$. Since it only needs a broadband pulse (with respect to the wavelengths), the LED model LED1550E from Thorlabs with a spectral FWHM of $100 \mathrm{~nm}$ (wide enough) applies very well due to its low-power consumption of only $2 \mathrm{~mW}$ [19]. This is especially important for 


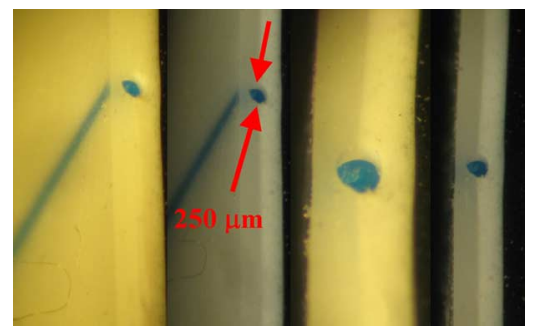

Fig. 3. A set of four photographs showing cross-section views of the layers configuration of a carrier prototype and the respective supported FBG.

the proposed portable application where it is provided a smaller power source composed by three class AA batteries each one with a voltage of $1.5 \mathrm{~V}$ (the same used for powering the microcontroller that controls the whole system).

\section{FBG Carrier}

A task to select a carrier material was done in order to increase the sensitivity of the FBG sensors to strains and at the same time for improving the adhesion. There are a lot of candidate materials for carrier usage; however, the one to be selected must also present an acceptable quality/price ratio for enabling its adoption by the industry. All of the former goals could only be achieved by using one of the three following thermoplastic polymers: the polyurethane (PUR) [21], the polyolefin (TPO) [22] and the polychloroethanediyl (polyvinyl chloride, or simply PVC) [23]. The polyurethane constitutes the option with best global performances. First, this material feels like real-leather, and second, it presents a very long durability and a high performance with regard to the flexibility. However, despite its advantages, the polyurethane is one of the most expensive flexible polymeric materials. Polyolefin can be a suitable alternative for the polyurethane by constituting a much lower price, but its flexibility and elastic limit are poorer than the case of polyurethane. Thus, in this sequence of ideas, the polychloroethanediyl was the selected material for use as FBG carrier due to its excellent performance/cost ratio and easy to manipulate during the manufacturing process. The polychloroethanediyl presents many other advantages when compared with its direct competitors, such as low production cost making this material highly competitive, high resistance to aging, high versatility, and simplicity of maintenance.

Fig. 3 shows the cross sections of the layers configuration of the carrier used for supporting the FBGs (a set of four photographs was taken under different directions and illuminations, in order to better illustrate the FBG sensor and the different layers of the carrier). The first layer of polychloroethanediyl (or external layer) is used to provide protection to the FBGs against external factors from the environment (e.g., mechanical chocks, scratches, corrosive atmospheres, among others). This is the visible layer after placing the carrier and it is about $200 \mu \mathrm{m}$-thick, which enables this layer to recover its initial shape after being easily bent. The optical fibers are flexible, can be easily bent and tend to recover their initial shape without breaking (when subjected to reasonable deformations, inside the safety limits). This means that a second layer (the middle layer) is needed to bond the FBGs to the carriers. The minimum thickness of this layer must be at least $300 \mu \mathrm{m}$ in order to ensure

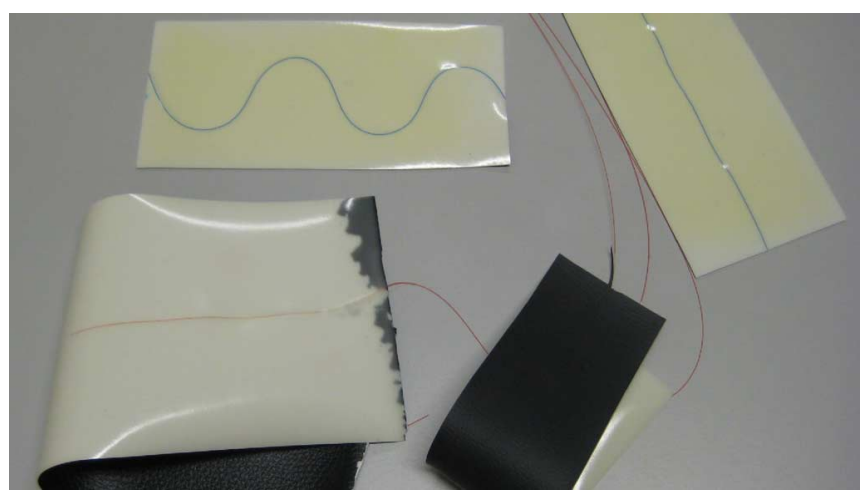

Fig. 4. A photography of some functional prototypes with FBG sensors already embedded on the respective carriers.

the full wrap of a fiber with a diameter of $250 \mu \mathrm{m}$. Finally, a third layer of polychloroethanediyl (or the inner layer due to obvious reasons) is the interface between the carrier and the wearable. The thickness of the inner layer is of major interest because it must provide a good transmission (enabling a good sensitivity) of cardio-respiratory stimulus and the environment temperature into the respective FBGs. Because the thickness of the inner layer constitutes a compromise between these physical measures, the most suitable thickness has been selected to be $400 \mu \mathrm{m}$. Fig. 4 shows some functional prototypes with FBG sensors already embedded on the respective carriers. Hydrogen loaded standard telecommunication fiber (Corning SMF28e+) was used for writing the gratings in these prototypes. Only the prototype with the optical fiber describing an undulated path is useful for sensing applications because it avoids early fiber breakage due to elongation. Thus, when compared with straight FBGs, the undulated FBGs allow for the successfully measuring of high strains. Further details about the embedding of FBGs into the PVC carriers can be obtained from the work done by Silva et al. [24].

\section{EXPERIMENTAL RESULTS AND DISCUSSION}

\section{A. FBG Displacement}

A Instron ${ }^{\circledR} 4302$ testing machine was used to evaluate the response of the FBG sensors before doing their integration into the carriers. In this sequence, an additional test was done by applying a continuous displacement at a constant increment of $16 \mu \mathrm{m} \cdot \mathrm{s}^{-1}$ and measuring the wavelength deviation. The graph of Fig. 5 reveals that the FBG sensors present a linear behavior for elongations above $0.6 \%$. The elongation range was limited to $1.6 \%$ in order to avoid breaking the fiber.

\section{B. Respiratory and Cardiac Frequencies}

The temporal strain variations of FBGs can be used to measure the respiratory and the cardiac frequencies as it is the case of previous works [25]. However, contrary to such a work, one of the innovations presented in this paper is the possibility offered to measure the respiratory and the cardiac frequencies with a single FBG sensor (since the two frequency regions do not overlap). In this context, the positioning of the FBG sensor is crucial in order to maximize the transmission of cardiac motions into the fiber. The secret relies in the carrier's shape, where its 


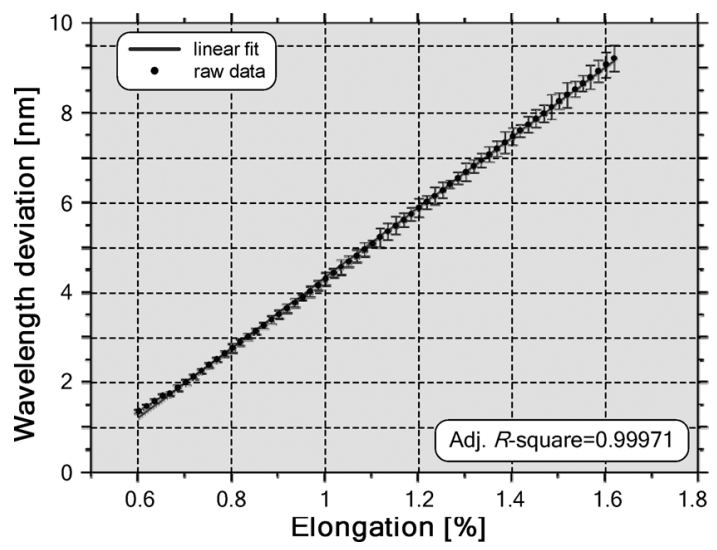

Fig. 5. The wavelength response to applied displacement.

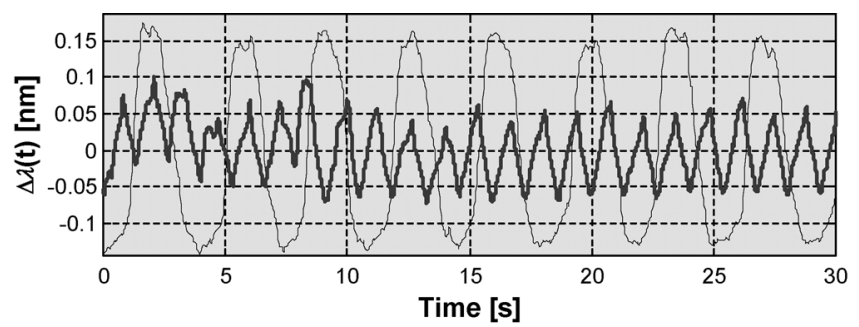

(a)

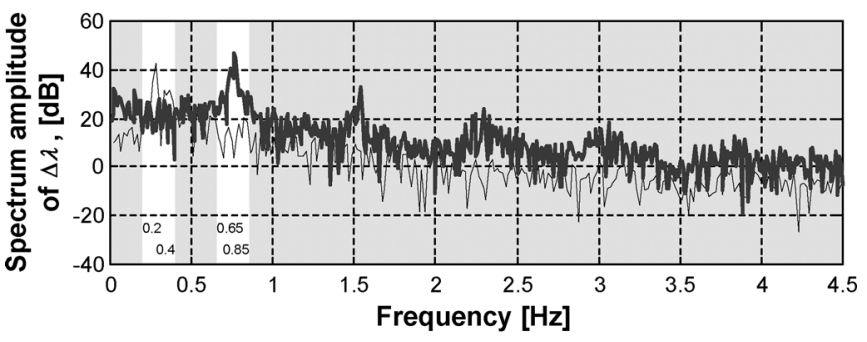

(b)

Fig. 6. Sensor response to a normal breath (raw data): (a) representation on time and (b) frequency domains.

structure must present enough area to cover a great portion of the chest and thus, in order to be capable for transmitting the chest movements due to the heart and respiration. For this purpose, it is expected that a wide rectangular structure to be more sensitive than a thin strap around the chest, because this enables the effect on the foil's corner to be sensed by the FBG sensor.

A set of tests were done with a group of 12 healthy subjects between 20 and 30 years old in order to evaluate the possibility to measure the heart and respiratory frequencies. Fig. 6(a) shows the wavelength shift, $\Delta \lambda(t)[\mathrm{nm}]$, sensor response to a normal (thinner line) and to a fast (thicker line) breath (raw data), where the external interferences do not appear to degrade the quality of the acquired signal - it must be noted that the wavelength shift is measured in relation to the wavelength, $\lambda_{0}$ [nm], of the strain-less situation, thus the (1) is $\lambda_{B}=\lambda_{0}+\Delta \lambda(t)$. The explanation for the observed small perturbations is mainly due to the transition between the inhale and the exhale stages. Fig. 6(b) shows the corresponding frequency spectrums, which reveals main frequency peaks between 0.2 and $0.4 \mathrm{~Hz}$ for normal breath and between 0.65 and $0.85 \mathrm{~Hz}$ for fast breath. An implementation in the digital domain makes easy the task to shift the central
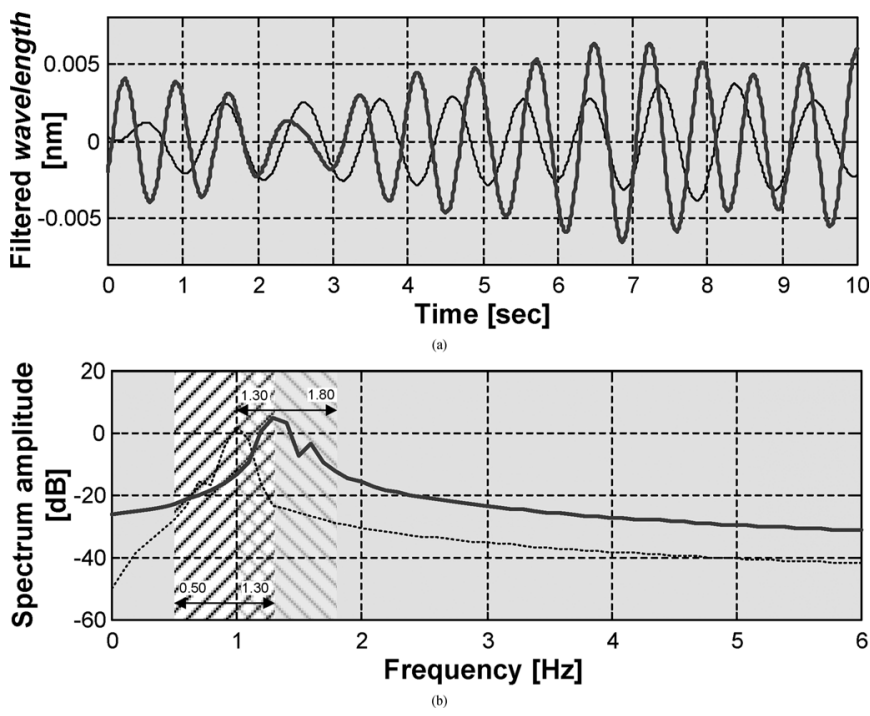

Fig. 7. (a) Developed sensing structure response with cardiac filtering stage and (b) respective frequency spectrums.

frequency of the filter and, consequently, retrieving the respiratory frequency.

The cardiac frequency can be obtained from the acquired FBG raw signal by applying a filtering. This task is relatively easy to do because the used optical detector allows the possibility to make fast Fourier transform (FFT) analysis and data logging. The acquired signals can be further processed by the analysis software, especially developed for this effect in the LabView graphical language. A bandpass filter for the $[0.2,0.4] \mathrm{Hz}$ frequency range allows the measurement of the respiratory component, while a second bandpass filter with a passband in the range $[0.5,1.3] \mathrm{Hz}$ can get the cardiac frequency. This second bandpass filter was used to discriminate the frequency set of interest around $1 \mathrm{~Hz}$, by cutting the respiratory components below $0.5 \mathrm{~Hz}$ and the high frequencies (e.g., the high-frequency noise) above $1.3 \mathrm{~Hz}$. Both passbands were implemented in the digital domain using the bilinear technique with the sampling frequency, $f_{s}[\mathrm{~Hz}]$, of $36 \mathrm{~Hz}$ for approximating their analog transfer function [26]. This performs well in situations of normal heart beats (the thinner line in Fig. 7), however, for higher cardiac frequencies this frequency range cannot be enough for subjects under effort doing high exercise. The thicker line plotted in Fig. 7 refers to a signal that was acquired under a condition of high cardiac frequency. Shifting the passband of the filter on range [0.5, 1.3] $\mathrm{Hz}$ to $[1.0,1.8] \mathrm{Hz}$ as postprocessing technique will make possible yet to still retrieve the cardiac component. Once again, this is not a problem because the filter is implemented in the digital domain, thus the coefficients can be updated in order to reflect the new transfer function.

Fig. 8(a) shows the respiratory signal after doing the bandpass filtering on the raw data acquired with the FBG sensor. The cardiac frequencies in Fig. 8(b) and (c) were, respectively, obtained by doing a second bandpass filtering on the raw data acquired with the FBG sensor and with the help of a Zephyr commercial sensor. The purpose of this last signal was validating the cardiac frequency acquired with FBG sensors and as it can be seen, 

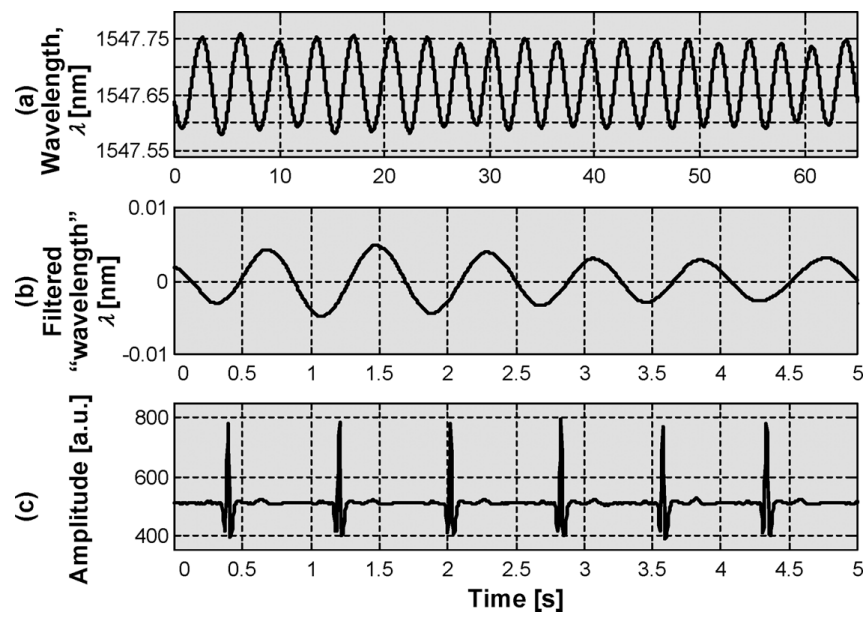

Fig. 8. Filtered signals: (a) the respiratory and (b) the cardiac frequency. (c) A cardiac frequency acquired with help of a Zephyr commercial sensor.

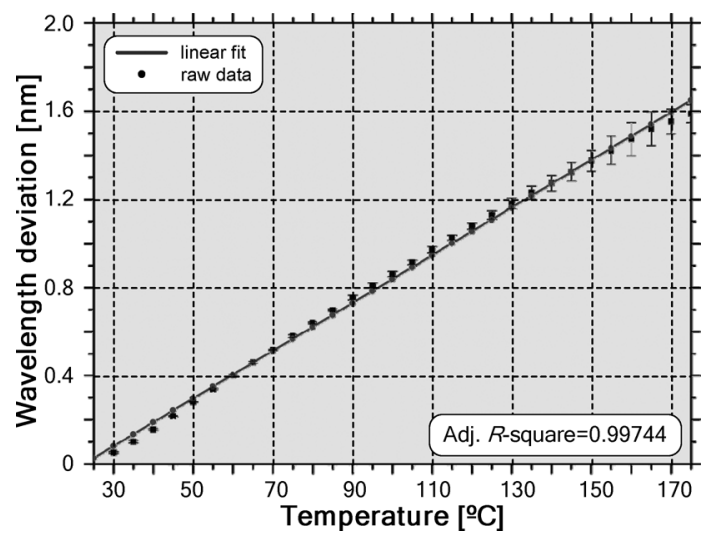

Fig. 9. The shift in the wavelength with reaction to temperature changes.

it exist a good agreement between the two waves. The vertical axis of Fig. 8(b) is expressed in terms of the wavelength (filtered "wavelength") because it was obtained by filtering the raw data (whose values are instantaneous wavelengths).

\section{Environmental Temperature}

Since two single- $\lambda$ FBGs can be combined with the help of the optical switch, the proposed photonic system contains an additional optical fiber sensor for sensing the environment temperature. In this sequence, the next step was the determination of the sensor performance when subjected to temperature changes. The thermal characterization was done by placing the FBG sensor of interest over a hot plate. The source of heat was programmed to provide temperatures up to $175^{\circ} \mathrm{C}$ at a rate of $1^{\circ} \mathrm{C}$ for each $3 \mathrm{~s}$. The FBG wavelength shift was recorded with the hot plate being heated. Fig. 9 shows the measured values, where it can be observed that the obtained data tends to present a linear behavior. The FBG sensor reacted to temperature changes at a rate of $0.1 \mathrm{~nm}$ per $1{ }^{\circ} \mathrm{C}$. The FBG sensor is not embedded into the carrier and kept floating with respect to the places where strains can happen due to the chest movements, in order to avoid the occurrence of cross-couples between the cardio-respiratory and the temperature components. Moreover, it is also important to have this FBG sensor fixed into a rigid support for avoiding coupling of undesirable strains resulted from the respiratory, cardiac and voluntary movements. This allows the measuring of the temperature in an independent way, when both the magnitudes of the respiratory and cardiac frequencies as well as the temperature vary simultaneously.

\section{CONCLUSION}

This paper presented a photonic system based on FBGs suitable for using in extreme harsh environments, as it is the case of fire scenarios where the temperatures are high. The novelty of the proposed solution over others available is the possibility to measure both the respiratory and the cardiac frequencies with a single FBG sensor. Moreover, the use of a digitally controlled optical switch allows the measurement of the environment temperature with a second FBG tuned at the same wavelength used for measuring the cardiac/respiratory frequencies. The interrogation system uses all-photonic components for doing the acquisition and is not restricted for the proposed functions. In fact, its scalability allows the inclusion of a larger number of FBGs. The polychloroethanediyl (PVC) material was used in the FBG carrier for increasing the sensitivity of the FBG responsible for measuring the cardiac/respiratory frequencies.

\section{ACKNOWLEDGMENT}

The authors want to acknowledgment that the integration of the FBGs into the carriers was done by TMG-Automotive (a company of the Textile Group, Têxtil Manuel Gonçalves $S A$ ) and the fabrication of FBG sensors was done by the FiberSensing Company SA.

\section{REFERENCES}

[1] D. Marculescu, R. Marculescu, S. Park, and S. Jyaraman, "Ready to ware," IEEE Spectrum, vol. 40, no. 10, pp. 28-32, Oct. 2003.

[2] P. Biswas, S. Bandyopadhyay, K. Kesavan, S. Parivallal, B. A. Sundaram, K. Ravisankar, and K. Dasgupta, "Investigation on packages of fiber Bragg grating for use as embeddable strain sensor in concrete structure," Sens. Actuators A: Elsevier Science Direct, vol. 157, pp. 77-83, 2010.

[3] A. Augousti, F. Maletras, and J. Mason, "Evaluation of cardiac monitoring using fiber optic plethysmography," Ann. Biomed. Eng., vol. 34, pp. 416-425, 2006.

[4] "The Georgia Tech wearable motherboard: The intelligent garment for the 21 st century," 1998.

[5] J. D. Jonckheere, F. Narbonneau, M. Jeanne, D. Kinet, J. Witt, K. Krebber, B. Paquet, A. Depre, and R. Logier, "OFSETH: Smart medical textile for continuous monitoring of respiratory motions under magnetic resonance imaging," in Proc. 31st Annu. Int. Conf. IEEE Eng. Med. Biol. Soc., Minneapolis, MN, Sep. 2-6, 2009, pp. 1473-1476.

[6] J. D. Jonckheere 1, F. Narbonneau, D. Kinet, J. Zinke, B. Paquet, A. Depré, M. Jeanne 1, and R. Logier, "Optical fibre sensors embedded into technical textile for a continuous monitoring of patients under magnetic resonance imaging," in Proc. 30th Annu. Int. Conf. IEEE Eng. Med. Biol. Soc., Vancouver, BC, Canada, Aug. 20-24, 2008, pp. $1473-1476$.

[7] A. Guillet, D. Kinet, J. Witt, M. Schukar, K. Krebber, F. Pirote, and A. Depre, "Optical fiber sensors embedded into medical textiles for healthcare monitoring," IEEE Sensors J., vol. 8, no. 7, pp. 1215-1222, Jul. 2008.

[8] S. K. T. Grattan, S. E. Taylor, T. Sun, P. A. M. Basheer, and K. T. V. Grattan, "In-situ cross-calibration of in-fiber bragg grating and electrical resistance strain gauges for structural monitoring using an extensometer,' IEEE Sensors J., vol. 9, no. 11, pp. 1355-1360, Nov. 2009.

[9] F. Xie, Z. Chen, and J. Ren, "Stabilisation of an optical fiber Michelson interferometer measurement system using a simple feedback circuit," Measurement: Elsevier Science Direct, vol. 42, pp. 1335-1340, 2009.

[10] H. Bao, X. Dong, C. Zhao, L.-Y. Shao, C. C. Chan, and P. Shum, "Temperature-insensitive FBG tilt sensor with a large measurement range," Opt. Commun., vol. 283, pp. 968-970, 2010. 
[11] T. L. Yeo, T. Sun, K. T. V. Grattan, D. Parry, R. Lade, and B. D. Powell, "Polymer-coated fiber Bragg grating for relative humidity sensing," IEEE Sensors J., vol. 5, no. 5, pp. 1082-1089, Oct. 2005.

[12] P. Orr and P. Niewczas, "An optical fiber system design enabling simultaneous point measurement of magnetic field strength and temperature using low-birefringence FBGs," Sens. Actuators A: Elsevier Science Direct, vol. 163, pp. 68-74, 2010.

[13] C.-L. Wei, C.-C. Lai, S.-Y. Liu, W. H. Chung, T. K. Ho, S. L. Ho, A. McCusker, J. Kam, and K. Y. Lee, "A fiber bragg grating sensor system for train axle counting," IEEE Sensors J., vol. 10, no. 12, pp. 1905-1912, Dec. 2010.

[14] J. Liu, C. Schmidt-Hattenberger, and G. Borm, "Dynamic strain measurement with a fiber Bragg grating sensor system," Measurement: Elsevier Science Direct, vol. 32, no. 2, pp. 151-161, Sep. 2002.

[15] D. Gurkan, D. Starodubov, and X. Yuan, "Monitoring of the heartbeat sounds using an optical fiber Bragg grating sensor," in Proc. 4th IEEE Conf. Sensors, Irvine, CA, Oct. 30-Nov. 3 2005, pp. 306-309.

[16] T. Erdogan, "Fiber grating spectra," IEEE J. Lightw. Technol., vol. 15, no. 8, pp. 1277-1294, Aug. 1997.

[17] Denselight Semiconductors. [Online]. Available: http://www.denselight.com

[18] FiberSensing. [Online]. Available: http://www.fibersensing.com

[19] Thorlabs GmbH, Germany. [Online]. Available: http://www.thorlabs.de

[20] Ibsen photonics. [Online]. Available: http://www.ibsen.dk/

[21] R. B. Seymour and G. B. Kauffman, "Polyurethanes: A class of modern versatile materials," J. Chem. Education, vol. 69, pp. 909-910, 1992.

[22] L. S. Lim, T. Harada, M. A. Hillmyer, and F. S. Bates, "High strength polyolefin block copolymers," Macromolecules, vol. 37, pp. $5847-5850,2004$

[23] Y. Saeki and T. Emura, "Technical progresses for PVC production," Progress in Polymer Science: Elsevier Science Direct, vol. 27, pp. 2055-2131, 2002.

[24] A. Silva, F. Gonçalves, P. Mendes, and J. H. Correia, "PVC formulation study for the manufacturing of a skin smart structure based in optical fiber elements," J. Polymers Adv. Technol., pp. 1-8, Dec. 2010.

[25] G. Wehrle, P. Nohama, H. J. Kalinowski, P. I. Torres, and L. C. G. Valente, "A fibre optic Bragg grating strain sensor for monitoring ventilatory movements," Meas. Sci. Technol., vol. 12, pp. 805-809, 2001.

[26] R. A. Losada and V. Pellissier, "Designing IIR filters with a given $3 \mathrm{~dB}$ point," IEEE Signal Process. Mag., vol. 22, no. 4, pp. 95-98, Jul. 2005.

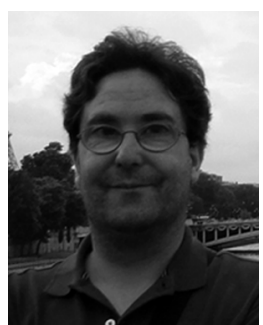

João Paulo Carmo (S'02-M'08) was born in Maia, Portugal, in 1970. He graduated in 1993 and received the M.Sc. degree in 2002, both in electrical engineering from the University of Porto, Porto, Portugal, and the Ph.D. degree in industrial electronics from the University of Minho, Guimarães, Portugal, in 2007. the Ph.D. thesis was on RF transceivers for integration in microsystems to be used in wireless sensors network applications.

Since 2008, he has been a Principal Researcher at the Algoritmi Center, University of Minho. $\mathrm{He}$ is involved in the research on micro/nanofabrication technologies for mixed-mode/RF systems, solid-state integrated sensors, microactuators and micro/nanodevices for use in wireless and biomedical applications. He is also involved with the supervision of Ph.D. students from the MIT-Portugal program.

Dr. Carmo is a Member of the IEEE Industrial Electronics Society.

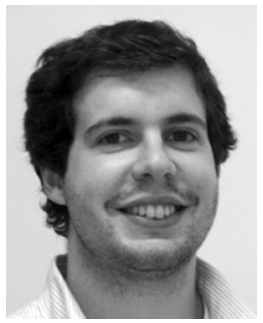

Alexandre Ferreira da Silva (S'10) graduated in 2007 in biomedical engineering (Integrated Masters) with specialization in medical electronics from the University of Minho, Braga, Portugal. Currently, he is working towards the Ph.D. degree at the Leaders for Technical Industries, University of Minho with the MIT-Portugal Program in the engineering design and advanced manufacturing focus-area.

Between 2006 to 2007, he spent six months at RWTH Aachen University, Germany, studying alternative sputtering processes in order to evaluate their performance and justify their utilization on electrodes production. In 2009, he performed an industrial internship at TMG Automotive for four months. In the same year, he was also a Visiting Student for four months at MIT's Materials Systems Laboratory.

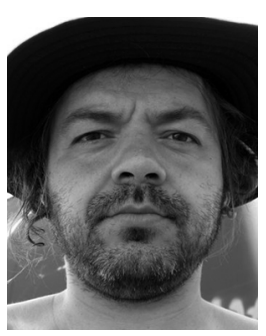

Rui Pedro Rocha graduated with a Degree in industrial electronics and computers engineering from the University of Minho, Minho, Portugal, in 2006 and received the M.Sc. degree working on dynamic simulation of the microchip encapsulation from the University of Minho in 2008. Presently, he is enrolled in the Doctoral Program at the Leaders for Technical Industries, MIT-Portugal in the engineering design and advanced manufacturing focus area . His Ph.D. thesis is dealing with the development of smart optical microsystems with sensing and actuating capabilities.

He spent six months in the Micro and Nanotechnology Centre, University of Oulu, Finland, working on the development of novel fabrication methods for nanoscale photonics and microelectronics components. During the past years, he did research in micromachining and microfabrication technology mainly in thermoelectric energy scavenging microsystems and solid-state, thin-film, rechargeable Li-ion batteries.

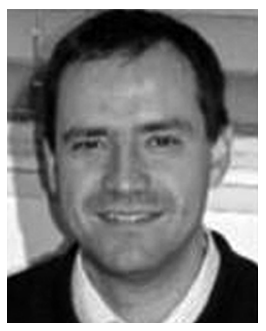

José Higino Correia (S'96-M'00) graduated with a Degree in physical engineering from the University of Coimbra, Coimbra, Portugal, in 1990. He received the $\mathrm{Ph} . \mathrm{D}$. degree from the Laboratory for Electronic Instrumentation, Delft University of Technology, Delft, The Netherlands, in 1999, working in the field of microsystems for optical spectral analysis.

Presently, he is a Full Professor with the Department of Industrial Electronics, University of Minho, Portugal. His professional interests are in micromachining and microfabrication technology for mixedmode systems, solid-state integrated sensors, microactuators and microsystems.

Prof. Correia was the General-Chairman of Eurosensors 2003 and MME 2007, Guimarães, Portugal. He is a Member of the IEEE Industrial Electronics Society. 